\title{
ALGUNAS NOTAS SOBRE LA FENOMENOLOGÍA DE LA HISTORIA EN EL PENSAMIENTO DE EDMUND HUSSERL \\ María Dolores Illescas*
}

RESUMEN: El efectivo acceso al conocimiento y comprensión de los diversos mundos culturales e históricos supone la constante apertura de un horizonte de co-humanidad y de su necesario correlato, el mundo uno y único, que es el mismo para todos. Esta apertura no puede darse, sino desde el presente, e hincará sus raíces en la constante tensión abierta entre lo actual y lo posible, o con otras palabras, en el abanico de posibilidades preseñaladas en cada caso por el entorno particular en que se vive.

\section{yose \\ REFLECTIONS ON THE PHENOMENOLOGY OF HISTORY IN EDMUND HUSSERL'S THOUGHT}

ABSTRACT: Proper access to knowledge and understanding of different cultural and historical environments supposes a constant opening to a co-humanitarian horizon and its essential cohort, the world, one and unique, but the same for everyone. This opening is only possible in the present harboring its roots in the constant tension between what is current and possible in the range of possibilities available in every situation in the environment in which one lives.

PALABRAS CLAVE: Husserl, fenomenología de la historia, sentido de la historia, multiplicidad de mundos culturales, fenomenología generativa.

KEY WORDS: Husserl, phenomenology of history, meaning of history, multiplicity of cultural worlds, generative phenomenology.

RECEPCIÓN: 9 de junio de 2014.

APROBACIÓN: 10 de noviembre de 2014.

* Facultad de Filosofía y Letras, UNAM/Universidad Iberoamericana. 
CITAM Derechos Reservados.

La reproducción total o parcial de este artículo se podrá hacer si el ITAM otorga la autorización previamente por escrito. 


\section{ALGUNAS NOTAS SOBRE LA FENOMENOLOGÍA DE LA HISTORIA EN EL PENSAMIENTO DE EDMUND HUSSERL}

\section{Introducción}

La experiencia del mundo en que vivimos tiene una estructura específica. Primeramente, se orienta, para cada uno, a partir de él mismo, y en tanto esto ocurre, ofrece al mundo como orientado de "aquí" a "allá", de lo próximo a lo lejano, del propio mundo circundante y familiar a los mundos extraños. Por otra parte, encontramos que la experiencia se halla igualmente orientada en cuanto forma parte de un mundo comunitario y ello por la manera en que, para mí, que soy sujeto de experiencia, los otros sujetos se presentan o me son dados; esto es, de manera mediata para mí (que percibo sus cuerpos vivos y desde esta base apunto certeramente a su vida de conciencia), aunque a la vez de modo inmediato para ellos mismos, o, con otras palabras, por empatía. ${ }^{1}$ Ahora bien, mediante estos otros, así como de su contribución a la formación de nuestro mundo vivido en común,

${ }^{1}$ Edmund Husserl, Zur Phänomenologie der Intersubjektivität (Texte aus dem Nachlass, Dritter Teil 1929-1935. Husserliana XV, Herausgegeben von Iso Kern), 1973, Den Haag, Martinus Nijhoff, p. 220 (en adelante citado como Hua XV). Lo anterior se explica porque la apercepción de sus respectivas vidas de conciencia (una especie de "apuntar a ellas", de captarlas de manera inevitablemente mediata, ya que jamás me serán dadas de manera originaria, como en cambio lo es mi propia vida), exige la base que ofrece la percepción de sus propios cuerpos vivos, de sus movimientos, gestos, posturas, etc., así como de la cualidad expresiva que se manifiesta en ellos, como "índice" certero de esa vida que me resultará siempre otra. A lo anterior ha de añadirse que, de acuerdo con Husserl, la empatía capta no sólo a los 
mis propias experiencias se enriquecen constantemente, se corrigen o complementan su sentido, lo cual ocurre en la medida en que nos referimos a las mismas cosas, como también por cuanto intercambiamos experiencias o transmitimos conocimientos sacados de nuestras respectivas experiencias, etc.

En esto, desde luego, juegan un importante papel las expresiones involuntarias, las que se pueden conocer de los otros por medio de su comportamiento corporal y las que (de manera aperceptiva) les adscribimos, por ejemplo en la captación de sus gestos como expresiones mediatas de su vida y, en ella, de la experiencia que naturalmente las acompaña. ${ }^{2}$ Pero a lo anterior han de añadirse las comunicaciones deliberadas y, en particular, las comunicaciones lingüísticas.

En su desarrollo, en parte activo, en parte pasivo (es decir, no voluntario, que simplemente acontece), la experiencia produce al conocimiento como una adquisición perdurable que permanece disponible en el recuerdo. E igualmente, ella esboza por anticipado lo que en adelante conviene esperar; es captación y aprehensión de lo nuevo según una serie de tipologías familiares que permiten reconocer (o recomprender), en alguna cosa que nos sale al paso, algo en cierto modo ya conocido. La experiencia del mundo es, por lo demás, siempre ya experiencia de la comunidad a la que pertenecemos, cualquiera que sea la extensión de esta experiencia y el sentido ampliado que en el ser unos-con-otros y unos-en-otros recibe el mundo mismo. La edificación orientada del sentido de ser del mundo, en la cual éste posee su acceso tanto como su modo propio de conservar su identidad, a pesar de la aparición de un sentido particular siempre nuevo, posee así una peculiaridad que es propia de cada mundo cultural. ${ }^{3}$

De tal modo, como sujeto de experiencia, tengo un mundo en torno próximo, el mundo familiar en el que soy y me siento "en casa" a partir de mi experiencia propia; se trata del mundo circundante bien conocido, íntimo, que adquiero de manera inmediata u originaria. A este mundo pertenecen también los otros que comparten conmigo el

hombres singulares, sino a comunidades humanas y, asimismo, a todas las configuraciones de la cultura.

${ }^{2}$ Ibid., p. 220.

${ }^{3}$ Ibid., p. 221. 
mismo entorno familiar, esto es, que tienen en el conjunto de experiencias adquiridas la misma cosa a partir de su experiencia propia. Y si bien cada uno posee lo que es para él familiar bajo un modo efectivo y originario, esto no quita que haya efectivas diferencias entre las experiencias de uno y otro, y por tanto, en la ulterior comunitarización de ellas. ${ }^{4}$

Husserl señala que la comunicación más originaria es explicitación (y en el mejor de los casos, expresión lingüística) de aquello de lo que he tenido directamente experiencia y de lo que el otro posee igualmente (en el caso más favorable) en su campo de experiencia, aun si éste no es actualmente presente $;{ }^{5}$ sin embargo, por el camino de la comunicación, también puede tomar parte en una experiencia que no posee de manera original, ganando así una experiencia secundaria (es decir, una experiencia del mismo contenido, aunque obtenida de otro que sí la ha tenido originariamente). Dicha experiencia posee su propio modo de confirmación a partir de la concordancia de la vida comunitaria, en la que se ligan cada experiencia original con las experiencias mediatas o secundarias, como también estas últimas entre ellas por la vía de los distintos tipos de comunicación, todo esto dejando subsistir las concordancias mencionadas en un desarrollo que se mantiene como válido. ${ }^{6}$

Aquel que comprende una comunicación, efectúa de inmediato por su cuenta la experiencia de índole intuitiva que le entrega lo comunicado "como si" fuera el otro, y "como si" él mismo lo viera, validando con ello la perspectiva que él también tendría de encontrarse justamente "allá", en la posición en la que se halla el otro, aunque también es verdad que los enunciados se comprenden, asimismo, de manera no directamente intuitiva, sino vacía (de intuitividad), como significados que no se ven cumplidos o plenificados por la presencia directa de

${ }^{4}$ Más aún, todas las cosas familiares, las que son conocidas e identificadas como tales por quienes comparten la intimidad del mismo entorno, no son verdaderamente tales sin que haya diferencias. Uno las conoce más precisamente, el otro menos, lo que es inevitable dado el "horizonte interno" de toda cosa o, con otras palabras, de su darse necesariamente en forma "perspectiva", por aspectos o perfiles (pero nunca de manera exhaustiva).

${ }^{5}$ Hua XV, p. 222.

${ }^{6}$ Ibid., p. 223. 
la cosa a la que aluden, lo cual ocurre por virtud de una lengua ya formada en relación con el mundo familiar y adaptada a su típica, que perdura de manera relativamente general. ${ }^{7}$ De manera que aquello que vale de manera objetiva para cada uno, puede decirse que vale para todos en la misma comunidad, por cuanto remite de un modo u otro a la fuente última de verdad que es la experiencia original de la cosa, como también, por otro lado, a la experiencia original de los otros.

Es, entonces, en la efectuación de la empatía, ya sea directa o indirecta, donde se confirma de manera continua que nosotros, los que participamos en ella, estamos referidos en nuestro intercambio mutuo al mismo mundo, que formamos parte de la misma humanidad familiar. Esto no excluye las correcciones recíprocas en el caso particular en que tengo que ver con individuos e igualmente donde se trata de las distintas comunidades de personas. ${ }^{8}$

Así pues, el horizonte del mundo se halla siempre en proceso de plenificación por la vía de experiencias que se corrigen y amplían recíprocamente, pero esto ocurre de una manera tal que el mundo guarda siempre una cierta apertura, una indeterminación que puede siempre admitir, aunque a título de esbozo, una nueva determinación experiencial más precisa o diferente.

Ahora bien, el conocimiento histórico se efectúa (no podría ser de otra manera), desde el respectivo presente de conciencia del historiador, como también de su propio mundo de vida, ${ }^{9}$ mundo que es para él efectivamente experimentado y experimentable. Desde aquí llega al preguntar retrospectivo sobre la humanidad pasada (o incluso actual) y el mundo vital que fue (o en su caso es) para estos hombres, precisamente manifestado y válido y por el cual fueron ellos motivados, para luego indagar, también retrospectivamente, acerca de la continuidad de la eficacia histórica del sentido que va pasando de generación en generación hasta llegar a nuestros propios tiempos y ofrecer, así, claves de suma importancia para la comprensión de nuestro propio mundo de cultura.

${ }^{7}$ Ibid.

${ }^{8}$ Ibid., p. 234.

${ }^{9}$ Pues, a fin de cuentas, el presente vivo es el "lugar" de determinación de todo sentido, sea actual o posible. 
Pero hay que advertir de inmediato que el historiador no limita su investigación ingenuamente al interior del mundo histórico que le es previamente dado. Su esfuerzo por hacer comprensible este mundo histórico particular se endereza también a descubrir, conforme a generalidad esencial, lo invariante en él. Puesto que lo singular en cuanto tal es propiamente impensable y por tanto indeterminable, se precisa el recurso a conceptos típicos que, por medio de sus diversos grados de generalidad, al menos apuntan a lo que es verdaderamente universal. ${ }^{10}$

De lo anterior se sigue que, en opinión de Husserl, la praxis propia del oficio de historiador tenga por tarea mostrar una historia universal en su pleno sentido, ya que ésta se extiende por encima de todas las formas del "nosotros", que se ven limitadas de continuo por las historicidades particulares. Mas esto sucede solamente en la forma de un "despertar" (o suscitar) continuamente al horizonte universal como una suerte de "plus ultra". A ello se debe que sea precisamente la humanidad histórica en su comprensión última el verdadero campo de trabajo del historiador y no los meros "hechos pasados". Recordemos aquí, así sea muy brevemente, que a todo yo (y a todo otro) pertenece un "nosotros" siempre abierto, en el sentido de que siempre va unido a él un horizonte de generalidad que tiene, a su vez, sus respectivas gradaciones, de acuerdo con lo que es conocido o desconocido, etc. Pero por ello mismo, sucede que el mundo histórico es para todos uno y el mismo mundo, aunque no sea sino en última instancia. Primero, como mundo circundante propio que no se reconoce como tal

${ }^{10} \mathrm{~A}$ la problemática que atañe a las diversas modalidades (o niveles) en las tipologías y, con ello al tránsito que va de la descripción de las experiencias y representaciones singulares o colectivas vigentes para un determinado grupo o sociedad, a los conceptos que recogen las configuraciones fundamentales de la vida espiritual, ya sea en el nivel de la generalidad empírica, o bien, en el de estructuras esenciales, Husserl dedica una parte significativa de las lecciones sobre Natur und Geist de 1927. Todo ello se encuentra en consonancia con la idea diltheyana de la abstracción de tipos en la historia a fin de salvar el escollo del relativismo. O también con la propuesta teórica de Georg Simmel, en cuanto a la necesidad de analizar la vida e interacción social al recogerla en formas invariables. En todo caso, la noción del "tipo" como una forma de generalidad todavía no conceptual resulta de mucho interés para las llamadas "ciencias del espíritu" debido a su concreción y también a su flexibilidad para guiar a modo de un esquema (que siempre puede modificarse) la integración de una multiplicidad. 
sino al encuentro con aquel otro mundo que le resulta extraño, pero a la postre abiertos ambos a la posibilidad de concordar en un horizonte más amplio que los abraza. ${ }^{11}$ Por eso, el universo de los hechos y procesos históricos que la historiografía tematiza y estudia, conforma nuestro constante horizonte histórico que incluye también lo desconocido o irreconocible que se mantiene, con todo, como accesible a un conocimiento posible. ${ }^{12}$

Así, la ciencia de la historia procede originalmente de la experiencia histórica y sólo es comprensible desde la vida misma, lo cual se deja ver con toda claridad en el hecho de que se expresa sin mayor problema en la lengua general, es decir, mediante un lenguaje que, al hallarse plenamente extendido sobre los cauces de la praxis vital, se adecua excelentemente a lo general y particular de las acciones y trabajos, las metas y finalidades, las pasiones y emociones humanas que en toda su riqueza y variedad estudia el historiador. Para Husserl, de lo que se trata es de mover el conocimiento teorético a la base firme de una observación concreta (aunque universal) de nuestra experiencia y mundo de experiencia, como también del reconocimiento de que, a fin de cuentas, nuestras ciencias todas derivan de uno u otro modo de este mismo mundo directo de la experiencia.

De tal suerte, el método histórico puede entenderse como extensión crítica y metódica de la experiencia natural, precientífica, que gira en torno a la ineludible exigencia de aclarar el propio ser histórico. Su meta consiste en desplegar el horizonte en el que las distintas memorias y tradiciones cobran sentido, llevándonos de tal suerte al ser de la vida pasada, con su respectivo mundo de vida anteriormente experimentado. Desde luego, la conexión con lo que fue vivido y percibido directamente por otros se da mediante de sus manifestaciones indirectas, alcanzando también los recuerdos a ellos comunicados. Y, ulteriormente, a

${ }^{11}$ Ciertamente, tenemos al mundo empírico comunitario como una unidad que incesantemente se va troquelando y corrigiendo de manera relativa, aunque a la vez abierta a lo infinito. Pero lo que sobre todo cabría destacar aquí es precisamente la tendencia a la unidad del ser "uno con otro", la cual, sin embargo, no se encuentra exenta de tensiones y discrepancias. Hua Materialen Band VIII: Späte Texte über Zeitkonstitution (1929-1934). Die C-Manuskripte (Herausgegeben von Dieter Lohmar), 2006, Dordrecht, Springer, p. 20-1.

${ }^{12}$ Hua XXIX, p. 243. 
través de la consideración de aquellas experiencias que fueron compartidas por muchos, así como de sus síntesis, que pueden ser cada vez más amplias. De tal modo, el dominio propio del historiador hinca su raíz en la experiencia y en la extensión de ella, y se despliega al seguir el hilo de las conexiones siempre más plenas y complejas al interior de la experienciabilidad realmente acreditable. Por ello, se dice que su ciencia es puramente descriptiva, aunque también echa mano de procedimientos inductivos que suponen la elaboración de cuidadosas comparaciones. Entonces, el auténtico sentido de la descripción en las llamadas "ciencias del espíritu" en general y de la historia en particular es para Husserl que ésta es descripción (sin menoscabo de un ulterior análisis y contextualización) de lo que fue en su día intuitivamente dado; y dado, a fin de cuentas, como mundo histórico cambiante.

A lo anterior hay que añadir que la ciencia puramente descriptiva es una ciencia que no excede la experiencia (aunque sí la eleva de nivel al tornarla en experiencia comprensora); por tanto, se mantiene en el espacio histórico humano que precisamente quiere conocer y el cual se acredita en experiencias convergentes como "auténtica" experiencia -ganada, en una primera instancia, sólo en tanto realidad relativa, aunque situada, a fin de cuentas, contra el horizonte de un tiempo ensanchado, que es "espacio vital" de la humanidad. ${ }^{13}$

Por contraste tenemos las modernas ciencias matemáticas de la naturaleza, donde, los rendimientos de la física, por ejemplo, se basan en que para ella todos los objetos de la naturaleza se determinan en una absoluta objetividad bajo la idea de un exacto ser "en sí". Y esto se logra mediante conceptos igualmente "exactos", es decir, geométricos o ideales y no ya de tipo empírico. Dichos conceptos proceden, pues, no de una abstracción directa desde la visión realmente experimentada, sino de un peculiar método de pensamiento: la idealización. De ahí que no puedan realmente ser vistos o tocados los puntos matemáticos, las rectas y demás objetos matemáticos que, para una física matemáti-

${ }^{13}$ Edmund Husserl, Die Krisis der Europäischen Wissenschaften und die Transzendentale Phänomenologie. Ergänzungsband (Texte aus dem Nachlass 1934-1937. Husserliana Band XXIX, Herausgegeben von Reinhold N. Smid), 1993, Dordrecht/Boston/London, Kluwer Academic Publishers, p. 313. En adelante citado como Hua XXIX. 
camente exacta, operan como punto de partida para procesos de aprehensión idealizadora. Entre la experiencia y la física descansa la idealización, y cuando la física se dice "ciencia de la experiencia" implica que si el tratamiento sobre la experiencia llega a ser exacto, necesariamente se debe a un manejo idealizador. Pero el problema aquí es, por un lado, el olvido de ese suelo fundante, el de la vida precientífica, desde el cual todos los conceptos científicos, por abstractos que sean, cobran sentido; y, por otra parte, el hecho de que las idealizaciones arriba mencionadas se proyectan en una infinitud que cae cada vez más y más lejos de lo que es efectivamente experimentable. Tales son las conocidas tesis defendidas por Husserl en La crisis de las ciencias europeas y la fenomenología trascendental.

\section{Lo que ha de entenderse por experiencia y mundo de experiencia}

En la experiencia tenemos al mundo, éste aparece. Sólo en tanto es experimentado podemos decir que el mundo existe; y sólo en la experiencia universal e intersubjetiva podemos decir que es válido objetivamente. Es cierto que la experiencia en su modo meramente intuitivo no es (todavía) objetiva en sentido pleno, pero esto no quita que sea el fundamento último incluso del mismo rendimiento cognoscitivo de la ciencia. Y no podría ser, en realidad, de otra manera, pues únicamente mediante la experiencia que de él tenemos, el mundo es lo que es para nosotros y gana un sentido concretamente determinado que, ciertamente, no equivale al de una vacía multiplicidad infinita. El mundo únicamente es tal para nosotros en cuanto pleno de sentido y en ello tiene una estructura mostrable intuitivamente, la que nos ata. ${ }^{14}$

Característica de la postura naturalista es, por contraste, la ceguera para todo lo que queda comprendido bajo el grueso título de la "inten-

${ }^{14}$ Pues no es arbitraria. E. Husserl, Natur und Geist. Vorlesungen Sommersemester 1927, (Husserliana Band XXXII. Herausgegeben von Michael Weiler), 2001, Dordrecht/Boston/ London, Kluwer Academic Publishers, p. 101. En adelante citado como Hua XXXII. 
cionalidad". ${ }^{15}$ Ceguera para la multiplicidad de fenómenos en los cuales, para mí y para todo yo en la "interioridad" de su propia vida psíquica, se da un mundo de experiencia. ${ }^{16}$ Por consiguiente, el pensamiento llega en verdad a carecer de fundamento (bodenlosen) cuando no parte del suelo que ofrece la plenitud de la vida enteramente concreta, con todo lo que ella conlleva, cuando no se sostiene sobre este suelo ni vuelve a él (en una especie de mirada retrospectiva) a fin de esclarecerlo, como ha de ser el caso de todas las abstracciones que derivan (o son sacadas) de dicha fuente.

Toda teoría y toda ciencia corresponde a la vida y se sostiene sobre el mundo preteorético, sobre el mundo de la pura experiencia, aquel en el que vivimos ante todo como hombres prácticos. $\mathrm{O}$, con otras palabras, toda ciencia, con la orientación propia de sus diversas tareas, remite necesariamente a algún modo de la subjetividad, ya que en realidad no puede sino ser relativa a ella. Y la subjetividad, tomada en su plena concreción, se nos ofrece como vida humana en el mundo, pero en un mundo que es, originariamente, intuitivo e histórico. ${ }^{17}$

Hemos de reconocer, entonces, que somos sujetos de un mundo que es siempre para nosotros un universo de realidades a las que, de una $\mathrm{u}$ otra forma, nos dirigimos intencionalmente; a las que, por así decirlo, nos hallamos volcados y que, en tanto tenemos o podemos tener experiencia de ellas, nos conciernen prácticamente, ya sea de manera positiva o negativa.

${ }^{15}$ De una manera muy general, puede caracterizarse la intencionalidad de la conciencia como un "dirigirse a" algo (precisamente aquello de lo que tenemos conciencia), o bien con la muy conocida expresión "toda conciencia es conciencia de..." (aunque el objeto o "contenido" del acto intencional sean, por ejemplo, las propias vivencias).

${ }^{16} \mathrm{Si}$ aplicamos esto al ámbito de lo propiamente histórico, encontraremos una interesante afinidad de la propuesta husserliana con la reflexión historiográfica que Robin G. Collingwood plasmó en los "Epilegómenos" de su famosa Idea de la historia (1985, México, Fondo de Cultura Económica, pp. 201-25). Esto se hace patente cuando el historiador inglés afirma que no existe una distinción entre el proceso o "hecho histórico" ("en sí”), que supuestamente vendría primero, y una manera especial de conocerlo, que vendría después (el pensar histórico); sino que, más radicalmente, el hecho histórico existe y adquiere validez como tal "sólo en y por la mente del historiador".

${ }^{17}$ En efecto, Husserl llega a afirmar con toda claridad que el mundo concreto, el "mundo de la vida" (Lebenswelt) no es otro que el "mundo histórico" mismo (Hua XXIX, p. 426). 
Así, aquello que es para cada uno un mundo existente es, respectivamente, el mundo de experiencia de la percepción actual, del recuerdo, de la pre-visión, con un horizonte de objetos mundanos conocidos aunque no se den (o presenten) actualmente. El término "experiencia" se refiere por tanto, al menos en una primera aproximación, a los modos subjetivos de aparición a los cuales debemos el sentido del mundo. ${ }^{18}$ Pero esto ocurre de un modo tal que en ello vale igualmente para mí y para cada uno, la experiencia de los otros. Mas aún, precisamente por cuanto tengo experiencia del mundo en común con otros, llego a nuestro mundo de experiencia, a nuestros fenómenos de este mundo que es el mismo para todos; llego asimismo a nuestra síntesis en la formación de una comunidad, a nuestros mundos circundantes experimentados en común como idénticos, a sus cambios, con sus unidades sintéticas en la puesta en común de nuestras propias percepciones o recuerdos, etc. ${ }^{19}$

Cada uno de nosotros tiene, pues, su mundo circundante y en comunidad tenemos un mundo en común; toda comunidad particular el suyo. Pero lo importante aquí es que en el cambio incesante al que se encuentran sometidos estos mundos, relativos y diversos, se conserva conscientemente una relación con "el" mundo como una unidad global constantemente presunta (pues no ha de olvidarse que el mundo tiene precisamente la estructura de un horizonte, de un límite que se desplaza). ${ }^{20}$

${ }^{18}$ Hua XV, p. 62.

${ }^{19}$ El concepto de experiencia se refiere, entonces, a lo dado - de manera evidente- en la captación sensible, inmediata, de objetos individuales; sin embargo, este concepto también abarca lo pre-dado pasivo (aquello que siempre hallamos como estando "ya ahí" previamente) y que supone aquella captación para tener lugar (piénsese, por ejemplo, en los datos sensibles o bien en todo lo que hemos vivido y desde los cual enfrentamos lo que es nuevo). En este último nivel de la vida de conciencia, verdaderamente fundamental, tenemos la "doxa" pasiva, pre-dada, como sustrato para las funciones cognoscitivas posibles que opera el sujeto, pero también como sustrato para todas sus valoraciones, planteamientos de metas prácticas y acciones. Véase al respecto, E. Husserl. Experiencia y juicio, 1980, México, UNAM, ed. de Ludwig Landgrebe, trad. de Jas Reuter, pp. 58-9.

${ }^{20}$ Para Husserl, en efecto, el ser del mundo como autocomprensible y permaneciendo igual desde el principio, tiene un carácter presuntivo. ¿Qué quiere decir esto? Para entenderlo, recordemos primeramente la presunción presente en toda experiencia (ya sea real o posible) de algún objeto mundano, de que el "mero lado" en el que lo experimentamos efectivamente, no es el objeto en su pleno ser él mismo. La intención experimentante nos lleva más 
Fijémonos ahora en el nosotros en su vida en comunidad y en tanto vida en el mundo (esto es, a la intersubjetividad como realidad puramente psíquica), así como también a los fenómenos que constituyen (o dan sentido) al mundo y que tienen lugar en esta vida. Al hacer esto encontraremos un tiempo inmanente (o tiempo propio de la vida de conciencia) común para este nosotros: un presente-para-nosotros, un pasadopara-nosotros, un futuro-para-nosotros que de ningún modo se reducen a una mera variante del tiempo del mundo. Cada uno, en la actualidad de su propia vida, encuentra esta intersubjetividad en la coincidencia de su presente con el presente de todo otro, y ello ocurre de tal suerte que en la síntesis propia de la formación de una comunidad, cada quien tiene su presente primordial u originalmente vivido (y en ello su temporalidad inmanente, su tiempo privado, por decirlo así). Pero todos estos presentes primordiales pertenecen al presente constituido intersubjetivamente, ya que ellos son presentes intersubjetivamente a la vez. Además, un mundo familiar (Heimwelt) común se constituye también intersubjetivamente, por virtud de este co-experimentar a la vez un mundo en común.

En lo tocante a la espacialidad, hay que decir que a pesar de no haber ningún "aquí común” que corresponda al "ahora común” a nivel

lejos de lo estrictamente actual a la presunción de lo todavía no experimentado, pero que pertenece al objeto mismo y puede ser captado verdaderamente en percepciones ulteriores, con lo cual una presunción avanza siempre otra vez en el sentido de un plus ultra. Por otra parte, este "siempre de nuevo" atañe también a los otros sujetos en sus respectivas experiencias, en la conexión experiencial de unos con otros respecto de las cosas que son las mismas para todos. En la experiencia aparece que con otros tengo mi objeto, que los otros también pueden recordarlo y volver a él siempre de nuevo e identificarlo de tal modo, e implícitamente con ello se da que lo mismo es perceptible y recordable para nosotros. Sin esto, sin este carácter esencial de ser para todos, no podríamos acceder a ningún objeto como existente; empero, la mera experiencia no da todavía un verdadero mundo (real) existente "en sí". Y, además, el ser del mundo es siempre todavía presuntivo ya que ningún modo del conocimiento del mundo puede estar perfectamente concluido. En cuanto a las presunciones científicas, éstas consistirían en determinados procesos intersubjetivos de creencia en la realidad del mundo presunto, mas precisamente en tanto infinitud por determinar, desde donde se perfila la idea-límite ideal, que es de suyo inalcanzable experiencialmente, de un conocimiento incondicionado (absoluto) cuyo correlato sería el mundo mismo en su pleno ser "en sí" (Hua XXXII, p. 236). 
originario, ${ }^{21}$ la intersubjetividad ha conferido siempre ya una cierta orientación al mundo existente, de manera que toda comunidad en su presente coparticipado tiene su espacio propio: la "residencia" para la familia, el "hábitat" para la ciudad, etc. El mundo aparece así con su forma espacio-temporal propia en los mundos circundantes intersubjetivos. La forma temporal objetiva, bajo la forma del tiempo inmanente intersubjetivo, del tiempo de la vida comunitaria; el espacio objetivo en los modos intersubjetivos de orientación en el espacio. ${ }^{22}$

"Pertenecen", entonces, al horizonte del mundo una forma y una típica universales que me son continuamente pre-indicadas desde mi propio mundo circundante. En las síntesis de identificación en las que cualquier cosa se revela mediante el proceso de su percepción, el cual me la entrega siempre por aspectos o perfiles que la van poniendo cada vez de manifiesto, adquiero al mundo (que es el horizonte "externo" de toda cosa), ${ }^{23}$ como sentido de ser. Lo adquiero con la conciencia de poder determinarlo explicitándolo de maneras muy diferentes y, sin embargo, identificándolo siempre de nuevo. La vía que tomo para identificar al mundo pasa necesariamente por el desvelamiento de otros egos y de su vida asimismo identificadora. ${ }^{24}$

${ }^{21}$ Conviene recordar aquí que el "punto-cero" de la orientación espacial, más aún, la vivencia que "abre" originariamente la dimensión del espacio, que es para cada uno siempre un espacio orientado, la ofrece la vivencia del propio cuerpo. Pero el cuerpo vivo de todo otro es precisamente otro cuerpo vivido originariamente (por él o ella) como propio, vivencia la cual no puedo alcanzar de manera originaria, pero sí aperceptivamente mediante la percepción de sus respectivos cuerpos como objetos del mundo, percepción la cual me remite, dada la expresividad propia de este peculiar "objeto", a la vida psíquica del otro, con lo que se revela la doble dimensión subjetivo-objetiva del cuerpo humano.

${ }^{22}$ Hua XV, p. 67.

${ }^{23}$ En la percepción de toda cosa del mundo se da siempre, junto a lo efectivamente captado de dicho objeto, una cierta anticipación de lo todavía no visto de esa misma cosa (esto es el "horizonte interno" de su determinación progresiva, abierta al infinito). Pero también, por otra parte, este pensamiento que anticipa "va más allá de esta misma cosa con todas sus posibilidades anticipadas [...] hacia otros objetos [que se dan con ella], aun cuando por lo pronto sean conscientes sólo en el trasfondo. Esto significa que toda cosa tiene no sólo un horizonte interno, sino también un horizonte externo, abiertamente ilimitado, de objetos que se dan junto con él' y cuyo plexo total constituye, precisamente, el horizonte del mundo (Experiencia y juicio, op. cit., p. 34).

${ }^{24}$ Hua XV, p. 201. 


\section{La sistemática del ensanchamiento de horizontes en el mundo de la experiencia}

Ahora bien, el mundo, a título de mundo único e idéntico para todos los que vivimos en una comunidad práctica, recibe un contenido siempre nuevo en cuanto mundo presente, en tanto nos es pre-dado. Su unidad es "correlativamente" la unidad de aquellos hombres que se encuentran unos con otros, de manera mediata o inmediata, en una cohesión de experiencia, de valores y finalmente como cohesión práctica, dado que este mundo es para todos ellos (para todos nosotros) un mundo circundante eminentemente práctico.

Por otra parte, este mundo familiar donde nos encontramos siempre ya, tanto como el modo conocido con el que anticipamos alguna cosa que adviene o cambia, tiene también un cierto "estilo" de dación (de ponerse de manifiesto) justo como mundo de nuestra experiencia. Y a este estilo pertenece igualmente la indeterminación de todo aquello que se halla implicado en dicho mundo, que afirmamos sin más como existente. ${ }^{25}$

Tocante a lo que cada vez es experimentado a partir de este mundo, sabemos qué estilo tendrá; conocemos el carácter típico de su finitud relativa, de lo que se puede abarcar de una mirada y en cierta forma ya conocemos, de aquello que está ahí para nosotros y que recibe nuevas formas en el marco de los caracteres típicos familiares. Nuestros tipos familiares desempeñan así el papel justamente de una tipicidad que nos permite registrar en objetos no típicos semejanzas en ellos y así comprenderlos o recomprenderlos. "Porque siempre proporcionan un mínimo 'núcleo de cognoscibilidad' que nos permite, aun cuando tratamos con objetos muy extraños, intentar la necesaria trasposición respecto del sentido de los objetos ya conocidos". ${ }^{26}$ Dicha tipicidad nos provee de un horizonte familiar de posible explicitación que mediatiza nuestras intuiciones, como también los efectos que podemos recibir, tanto de la acción de otros sujetos, como de objetos culturales. ${ }^{27}$

${ }^{25}$ Ibid., pp. 197-8.

${ }^{26}$ Roberto Walton, "Transmisión de sentido y nexo de eficacia" en Escritos de filosofía, núm. 43, 2003, p. 373.

${ }^{27}$ Ibid. 
Lo que hay que subrayar aquí es que el mundo siempre llega más lejos que nuestra experiencia presente; en tanto multiplicidad finita, es siempre un mundo conocido, pero también un mundo que se ensancha en la extensión de la experiencia que vamos adquiriendo de él y que siempre permanece susceptible de irse ampliando más. Para ello puedo recurrir a los otros, puedo interrogar a quienes me son cercanos a propósito de su mundo circundante (este mundo que para mí es indeterminado en tanto es el mundo que ellos han experimentado, pero que permanece para mí desconocido en sus particularidades, etc.). En todo lo cual ocurre que incluso el estilo -como estilo de ampliación del conocimiento-, se anticipa en lo tocante a las esferas mundanas desconocidas; se anticipa justamente como un estilo reiterable.

Más aún, en su horizonte reiterable de futuro, el mundo es reiterable igualmente bajo la forma de una ampliación que debe continuar. Esta potencialidad pertenece también a nuestro pasado, puesto que siempre hubiéramos podido aprender a conocer nuevas cosas y en tal dirección más bien que en tal otra, de la misma manera que ahora mismo la prosecución de su ensanchamiento tiene abierto un abanico de posibilidades y direcciones diversas. ${ }^{28}$ Así, el mundo se temporaliza en el flujo de la temporalización subjetiva e intersubjetiva, pero la potencialidad inherente a la intersubjetividad de ampliar su conocimiento en un sistema fundado lleva en sí el carácter de ser una potencialidad reiterable; de tal modo, el mundo se expande y nuevas cosas que en absoluto estaban allí llegan a ser tomadas en consideración. Por otra parte, además de la tipicidad en la edificación de cada mundo circundante relativo en su infinidad abierta propia, cada uno de dichos mundos posee su propio estilo de lo lejano, indicando de manera indeterminada proximidades que, al menos por el momento, permanecen inaccesibles. ${ }^{29}$

Cada mundo familiar posee, por tanto, una apertura propia por la cual su conservación continúa manteniendo su identidad como tal mundo

${ }^{28}$ Hua XV, p. 199.

${ }^{29}$ Ibid., pp. 219-20. En este sentido, "la cercanía prescribe ciertamente el estilo de lo lejano" (Hua XXXII, p. 116). 
familiar, precisamente, y su extensión espacio-temporal aparece posible. A este mundo pertenecen también otros sujetos que nos son muy próximos y tienen las mismas cosas que nosotros a partir de su propia experiencia. E igualmente se incluye allí el intercambio inmediato de experiencia en el que nace el entorno familiar común, pero ahora en un sentido ampliado.

\section{El encadenamiento generativo y su "infinitud"}

A la formación del sentido del mundo objetivo, por cuanto se dice de él que es "infinito", pertenece la obra y acción de la intersubjetividad generativa (es decir, aquella que da lugar a la liga y sucesión de las generaciones), puesto que cada uno de nosotros debe ampliar este mundo en su temporalización finita, aun si es solamente de manera finita; en efecto, el encadenamiento de las generaciones entre sujetos se halla constituido, para cada uno, reiterativamente a partir de la finitud. Y la vida y su temporalización se cumplen en una presunción continua que se reitera, a saber, la presunción de que aquellos que son otros para mí, tienen el mismo mundo en torno finito que yo y además un mundo circundante que yo no tengo todavía o que no tengo en absoluto y no puedo tener fácticamente; o bien, que mis otros tienen, a su vez, sus otros y éstos últimos sus respectivos otros, etc., y en esta serie de mediaciones, gente que llegue a serme fácticamente inaccesible. ${ }^{30}$

Con todo, siempre espero poder relacionarme con quienes no conozco todavía y tengo experiencia de los otros como detentando estas mismas posibilidades. Lo cual se hace, por lo demás, en un encadenamiento generativo, por lo que el mundo en su temporalidad sucesiva va explicitando su validez de ser en el encadenamiento abierto de la comunicación; desde luego, con esto se funda también la posibilidad de comprender el mundo como un mundo de la vida de generaciones que se suceden unas a otras en la unidad de una tradición histórica, luego, como un mundo cultural histórico; y enseguida tendríamos

\footnotetext{
${ }^{30}$ Hua XV, p. 199-200.
} 
la posibilidad de explicar el mundo y la humanidad en una temporalidad abierta.

Sin duda, en el proceso de ir desplazando los límites de nuestro mundo, tiene una importancia fundamental el encuentro con los extraños y su respectivo mundo de vida, que es también un mundo familiar, pero ciertamente otro diferente del propio. Mas, ¿qué ocurre con "el” mundo que, como hemos visto, no nos es dado sino de manera "fragmentaria", como un mundo de la vida meramente finito? El mundo que existe para nosotros es siempre, en tanto horizonte, mundo de una experiencia posible; pero esta posibilidad, ¿no quiere decir ante todo un acceso potencial (luego una verificación) en términos de tiempo y espacio? En lo tocante a la temporalidad, tenemos la generación -mis padres, nuestros padres, los padres de mis padres, etc.-, es decir, un pasado pre-indicado como aquél de los mundos circundantes que pertenecieron a nuestros antepasados, pero que valen para nosotros, por poco conocidos o indeterminados que sean, en la anticipación que podemos hacer de ellos y su posible conocimiento, con lo cual se forma el horizonte abierto de lo que puede anunciarse históricamente. ${ }^{31}$

Es claro que en todo esto tenemos una limitación general que vale de antemano, y es el hecho de que en este mundo del nacimiento y la muerte tengo una vida temporalmente finita y fuerzas finitas, aunque siempre en desarrollo; en este marco, encuentro que tengo un cierto dominio de lo lejano más externo, que existe para mí al mismo tiempo que para aquellos que comparten mi mundo familiar. Alcanzar este dominio de lo lejano no se da, empero, sin producir de nuevo las apariciones propias de otro dominio de lo lejano. Pero estas apariciones no indicarán ya objetos de una experiencia fácticamente posible (vermöglich); en otros términos, estos objetos no podrán ser ya pre-indicados por estas potencialidades fácticas como existiendo efectivamente; así ocurre para todos nosotros, aunque viajemos y ensanchemos con ello nuestro mundo. En todo caso, el mundo permanece como el mundo de tal potencialidad de la experiencia, pero sobre todo como aquel que, a partir de allí, es para nosotros siempre en la finitud.

${ }^{31}$ Ibid., p. 206. 


\section{Devenir y síntesis de mundos circundantes}

Es importante notar en este punto que la síntesis de los diversos mundos de la vida, tanto como de las generaciones que se suceden, no produce, como parecía en una primera aproximación, un mundo verdaderamente infinito como "mundo de una experiencia posible", entendiendo por esto el universo de los entes que la humanidad, en una comunicación universal y en tanto generativa (es decir, que comprende los lazos entre diversas generaciones), con su tiempo histórico, pudiera efectivamente experimentar ad infinitum todas las ampliaciones potenciales de su entorno; el sentido mismo de ser del mundo se halla fundado, ciertamente, sobre el sentido "otro trascendental" (esto es, constituyente de sentido), pero de un modo tal que conduce siempre de nuevo a una esfera mundana finita. ${ }^{32}$ Lo anterior implica, claro está, la dinámica de la ampliación del mundo familiar y en ello la aparición, al menos presuntivamente, de cada vez otros y nuevos otros sujetos por venir... Y también, por otra parte, que el mundo valdría siempre para todos los nuevos sujetos, aunque eventualmente se le tuvieran que hacer correcciones a la serie de determinaciones que sobre él se hubieran ya ganado.

¿Cómo opera este proceso de una constitución progresiva del mundo, de una formación progresiva de lo propio y lo extraño con sus mundos circundantes que cambian en este proceso y que son para sí en tanto valen para sus respectivos sujetos como mundos vivos, que reciben de estas personalidades una forma siempre nueva, un sentido teleológico o afectivo siempre nuevo? ¿Cómo ocurre que a partir de mí -y de nosotros- se constituye una pluralidad de "humanidades" como pueblos, que con toda su irreductible diversidad, forman sin embargo una humanidad única?

Cada una de estas "humanidades" en plural tiene como mundo circundante simplemente un mundo, pero un mundo que tiene su concordancia propia. En el carácter universal de la realidad, éste no es, sin embargo, un mundo concordante de antemano con el que vale para

${ }^{32}$ Ibid., p, 207. 
otros grupos humanos, para otras culturas o sociedades. ${ }^{33}$ Lo que en todo caso permanece como constantemente común es la estructura natural con su humanidad, que debe entenderse en el sentido más universal; esto no quiere decir que para mí y para nosotros en nuestro grupo familiar, como también para todo otro, el respectivo mundo cultural se halle pre-levantado sobre una naturaleza universal pura. ${ }^{34}$

Pero, entonces, ¿qué es aquello que permanece válido para mí, en vista de la discordancia que existe entre mi (o "nuestra") experiencia y la de otros grupos humanos? Hasta aquí, señala Husserl, hemos aceptado como estados de hecho en el mundo a las sociedades y culturas extrañas. Mas en esto no ha habido síntesis, en el sentido de que nuestro mundo circundante simplemente incorporara lo que para los extraños es válido. Mi mundo "ampliado" no se ha constituido de un modo tal que fuera simplemente una amalgama de mi mundo con los otros mundos, formando todos ellos una unidad extraída de la convicción en la experiencia concordante, que sería propia de todos los hombres de tal círculo ampliado. ${ }^{35}$

El mundo que vale para mí y para nosotros a partir de la concordancia de nuestra experiencia tiene un horizonte espacio-temporal, el horizonte posible del ser real; sin embargo, este ser no debe, como hasta el presente, ser anticipado solamente bajo la forma de una continuación abierta en el estilo que ha prevalecido hasta ahora, es decir, en una experiencia efectivamente posible. En tal experiencia, los hombres en general se anticipan o aperciben siempre como hombres que pertenecen a mi tipo de humanidad, con lo que "el" mundo recibe demasiado pronto la forma de $m i$ mundo en torno propio, el que me es familiar. Empero, en cuanto recibe una determinación diferente, el horizonte espacio-temporal real y abierto se reconoce como ocupado por un pueblo extraño con su mundo circundante extraño, distinto del mío,

${ }^{33}$ Ibid., p. 216.

${ }^{34} \mathrm{Sin}$ duda, estas diferentes humanidades pertenecen a un solo y mismo mundo que es en primera instancia el mundo para todas ellas. Pero esto ocurre ante todo bajo el modo de un mundo de realidades que se prosigue y continúa prosiguiéndose con un "nosotros" siempre nuevo y siempre con nuevos "nuestros mundos".

${ }^{35}$ Hua XV, p. 216. 
por donde se distinguen luego el mundo real en general y el mundo circundante, que pertenece a un pueblo determinado.

Lo que hay que tener muy claro es que el horizonte de ser para los otros es solamente conocido bajo la forma del dominio extraño y de la comprensibilidad (no siempre fácil) de su mundo en torno. Mundo que se halla solamente indicado y que sólo puede explicitarse de manera indirecta a partir de aquello que se puede comprender de la forma más general de su cultura. ${ }^{36}$

Atendiendo a lo anterior, ¿cómo puede hablarse de un mundo de experiencia que vale para todos los hombres en general, ya sean conocidos o desconocidos? Al aprender a conocer, por una empatía bien lograda, ${ }^{37}$ aquello que me resulta extraño en un pueblo y en su mundo circundante, llego a un mundo de estados de hecho ampliados. Y el mundo de estados es cada vez diferente para los distintos círculos de personas al interior de un pueblo; esto ocurre de tal manera que cada uno sabe, al unísono con todos los demás, que tiene experiencia de un solo y mismo mundo, aunque cada grupo humano particular lo aprehenda de manera diferente. ${ }^{38}$

¿Cuál es la base de esta mismidad? ¿Cuál la experiencia universal concordante a la que corresponde un mundo auténticamente universal? Sin duda poseemos el mundo abierto sin fin tras sus realidades efectivas conocidas y sus posibilidades desconocidas, cada uno a partir de sí, y también por la vía de las comunicaciones cuyas redes soportan la comunidad humana de experiencia. Nuestro mundo circundante, aquel que se liga a nosotros de manera histórica a partir de nuestros respectivos presentes, el mundo progresivamente accesible, posee el horizonte abierto de una naturaleza tal que trasciende la naturaleza que pertenece a este círculo y nos es efectivamente accesible.

Con todo, conviene recordar aquí que no poseemos el mundo, sino en el modo del horizonte y en realidad no poseemos todos los mismos hechos efectivamente dados, sino sólo como posibilidades abiertas. Así,

${ }^{36} \mathrm{Ibid}$, p. 217.

${ }^{37}$ Aunque ha de aclararse en seguida que ésta seguirá siendo siempre limitada.

${ }^{38}$ Hua XV, p. 217. 
estamos lejos de poder tener experiencia en algún momento ulterior, de manera fáctica, de todo aquello que otro hombre vive en sí como hombre fáctico; tampoco cabe duda de que no todos los hechos nos son accesibles en el ejercicio de nuestros poderes efectivos. ${ }^{39}$

Por lo demás, el mundo no es el único horizonte general con el que nos encontramos, sino que las realidades individuales de las que ya tenemos experiencia tienen sus horizontes internos (pues se nos dan sólo perspectivamente) y dichos horizontes no son de ningún modo perfectamente accesibles, y lo mismo aunque el mundo de la experiencia sea un universo abierto de realidades concretas que podemos percibir, recordar, etc. En esta medida, tenemos todos un cierto acceso (al menos de manera indirecta y por mediación de los otros con quienes entramos en contacto), a todas las realidades a las cuales ellos tienen acceso también, como objetos idénticos de una experiencia posible, sigue siendo verdad que ni ellos ni nosotros tenemos acceso a todos los rasgos objetivos de dichas realidades. ${ }^{40}$

Ahora bien, eso que yo aprendo a conocer en mi experiencia concordante por cuanto ella determina para mí un mundo ya válido, eso mismo concierne al mundo del que otro tiene también experiencia, puesto que él es reconocido por mí como referido al mismo mundo. Yo no tengo experiencia de este otro sino en tanto él experimenta todo aquello de lo que yo tengo o puedo tener experiencia, de manera que un suelo de comunidad efectiva por empatía se da como dominio de accesibilidades efectivas comunes. Tenemos experiencia, pues, del mismo ámbito de realidades $\mathrm{y}$, en particular, compartimos la experiencia de lo real que nos concierne. Que el otro no posea ciertas experiencias y que de hecho no pueda en absoluto poseerlas no perturba la concordancia intersubjetiva de nuestro mundo común. ${ }^{41}$

Comprender al extraño como sujeto de su mundo familiar y ligado a su grupo de pertenencia por una comunidad concordante de experiencia; apercibirlo así, es una experiencia con un horizonte todavía muy inde-

${ }^{39}$ Ibid., pp. 228-9.

${ }^{40}$ Ibid., p. 229.

${ }^{41}$ Ibid., p. 230. 
terminado. Pertenece a la experiencia efectiva del otro y a su comprensión explícita, a la producción completa de una comunidad de experiencia con él, aprender a conocer su mundo familiar que es para él cada vez presente y con ello el grupo humano que le es asimismo familiar; esto es, su acción efectiva, su creación en su mundo circundante, la forma en que este mundo familiar ha recibido y continúa recibiendo su sentido. El problema radica en saber hasta qué punto puedo adoptar por mi cuenta las vigencias de experiencia de los otros, de los extraños, para luego proceder a una síntesis de su mundo familiar con el mío. ${ }^{42}$

Ciertamente, y debido al elevado nivel de generalidad en el que se pone en juego la tipicidad del horizonte familiar, las analogías que desde él se articulan prefiguran posibles líneas para un conocimiento más preciso que, por medio de un permanente proceso de corrección, puede avanzar hacia ulteriores determinaciones o incluso hacia modos distintos de determinación. Pero, ¿es el mundo una mera síntesis progresiva de finitudes históricas?

La cuestión aquí no sería, desde luego, la de saber quién, en su inmanencia de experiencia, tiene la precedencia, sino lo que hay tocante a la comunidad de experiencias de los dos lados en su síntesis de concordancia, posible o por producir. Si ella se produce, e incluso en síntesis con grupos humanos siempre nuevos, siempre se puede todavía preguntar por el carácter definitivo de esta concordancia o, más bien, reconocer que dicha concordancia es siempre de nuevo relativa y en movimiento. Al comprender las experiencias y las concordancias de los otros, teniendo a la vista no ya al mundo como mundo de mis convicciones privadas, sino al mundo para todos, las dejo valer en tanto ellas co-constituyen mi mundo. Esto implica tener en cuenta a los otros como susceptibles de co-validar mis propias vigencias de experiencia; con ello gano la posibilidad de circunscribir un mundo posible de experiencia, como mundo circundante de mis semejantes que se mantienen en la unidad de una comunidad de experiencia, pero también una humanidad en general, es decir, en la infinidad abierta. ${ }^{43}$

${ }^{42}$ Hua XV, p. 233.

${ }^{43}$ Ibid., p. 234. 
Demos ahora el "salto" al nivel trascendental, dejando así el nivel de los meros hechos, para preguntar: ¿cómo se pueden llevar las experiencias y concordancias de experiencia de todos los co-sujetos trascendentales (o dadores de sentido), a una concordancia que habría de proseguir indefinidamente, en la cohesión posible de todas ellas? ¿Cuál sería, pues, la forma esencial de una concordancia absoluta de la experiencia intersubjetiva que se extiende al infinito, a través de los mundos familiares y culturales relativos y sus síntesis?

\section{Caminos abiertos}

Se abren aquí dos posibles vías para continuar la indagación que se ha venido bosquejando:

1) Respecto de la naturaleza infinita que se extiende más allá de la naturaleza efectivamente accesible que pertenece a cada mundo circundante, es posible afirmar que poseemos, en efecto, tal naturaleza "infinita", pero como la idea de una "verdad en sí" por alcanzar. ${ }^{44}$ Con ello se tiene la meta que orienta los esfuerzos de la ciencia natural. ${ }^{45}$ 2) En lo tocante al mundo uno y único, considerado esta vez como correlato de un "nosotros" universal que enmarcaría las diversas comunidades históricas particulares, tenemos que se articula sobre todo por virtud de una forma final que, al interior de una estructura universal de horizonte, no permite más que una determinación por aproximación. En dicha teleología tendríamos, por tanto, el sentido unificador de la pluralidad de historias particulares y sus respectivas vigencias, pero como tarea por realizar. Tarea de índole eminentemente ética: a saber, la de un proceso, por otra parte siempre amenazado, de humanización del mundo. Este proceso unificador de la historia es, ante todo, un constante advenimiento de sentido.

${ }^{44}$ Para Husserl, en efecto, la verdadera naturaleza como unidad sintética de todas las experiencias de la humanidad es una idea que, precisamente, guía la ciencia de las "idealizaciones", siendo éstas fundamentalmente de índole matemática (Hua XXXII, p. 73).

${ }^{45}$ En vista de lo anterior, también cabe formular el problema que aquí nos ocupa en términos de la coexistencia, o de la posibilidad de una infinidad de mundos familiares en conexión mediata, en relación con el problema de la constitución de una tal naturaleza infinita. 
Quisiera añadir a lo anterior que la descripción del mundo circundante intuitivo, de suyo concreto, fáctico y singular, da lugar a la formación de "conceptos de experiencia" o tipificaciones empíricas. Más aún, la generalidad que ganamos gracias al conocimiento del entorno individual concreto no es menos individual que aquella de los objetos singulares descritos en sus particularidades. Ya cuando en la vida práctica avanzamos de experiencia en experiencia, de un objeto singular a otro, nos vemos empujados a la generalidad y, querámoslo o no, cuando una nueva singularidad se nos presenta, la experimentamos en su tipo individual. ${ }^{46}$ Lo general descansa, así, en la forma de apercepciones típicas, levantadas sobre la individualidad determinada del mundo circundante en que vivimos; por eso, esta generalidad puede decirse con todo derecho individual, por cuanto se sostiene sobre tal entorno concreto.

Ahora bien, las ciencias culturales o históricas son todas ellas ciencias del mundo circundante y se sostienen en el marco de la accesibilidad concreta-intuitiva, directa o indirecta, del mundo que es, en cada caso, el escenario para la praxis humana. Ciertamente, el mundo fáctico individual tiene sus respectivas estructuras, y las tiene con necesidad esencial, pero ellas le pertenecen sobre todo en cuanto unidas a un mundo posible, por lo que estas estructuras necesarias ya no se hallan especialmente ligadas al factum particular que llamamos mundo circundante intuitivo; de ahí la distinción ulterior entre mundo circundante (Umwelt) y mundo en sentido pleno (Welt), que señala una estructura apriórica general fundada en la esencia de una posible experiencia y mundo de experiencia, pero al llegar a este último punto, nos salimos de la esfera propia de las ciencias de la cultura.

Consideremos ahora, de nueva cuenta, el mundo de experiencia logrado por una comunidad de diversos sujetos y ordenado como el mundo de sus concreciones intuitivas reales y posibles. Las estructuras presuntivas más amplias de dicho mundo, aquellas que pertenecerían al mundo tomado en sentido pleno, quedan todavía por alcanzar, aunque valen ya como existentes. Y, sin embargo, con ello tenemos también el índice que apunta a una absoluta intersubjetividad idéntica como correlato de un mundo que es, a fin de cuentas, el mismo para todos.

${ }^{46}$ Ibid., p. 200. 
CITAM Derechos Reservados.

La reproducción total o parcial de este artículo se podrá hacer si el ITAM otorga la autorización previamente por escrito. 